\title{
Correction to: Design of Heterogeneous Evaluation Method for Redundant Circuits
}

Huicong Wu, Jie Yu, Yangang Wang, and Xiaoguang Wang

\section{Correction to:}

Chapter "Design of Heterogeneous Evaluation Method

for Redundant Circuits" in: M. Qiu (Ed.):

Smart Computing and Communication, LNCS 11344, https://doi.org/10.1007/978-3-030-05755-8_40

In the original version of this chapter, a wrong project number was stated in the Acknowledgements Section. This has now been corrected. 